\title{
Modeling and Implementing Compressible Isotropic Finite Deformation without the Isochoric-Volumetric Split
}

\author{
Fuzhang Zhao \\ APD Optima Study, Lake Forest, CA 92630, USA \\ Email: fuzhangzhao@yahoo.com
}

\begin{abstract}
Constitutive models and finite element implementations of compressible finite deformation are straightforwardly formulated by the general isotropic continuum stored energy (CSE) functional without the isochoric-volumetric split. Coupled stress and elasticity tensors in reference and current configurations are derived. Modeling and predicting capabilities of the general CSE functional are exhibited through multiaxial experimental tests of compressible NR and SBR rubbers. Characterization of kinematic relation, rather than pressure-volume relation, is emphasized in experimental tests of compressibility. The isochoric-volumetric split does not hold based on either theoretical analyses or experimental validations.
\end{abstract}

Keywords: Compressible finite deformation, finite element implementation, isochoric-volumetric split, Poisson function, work-conjugacy.

\section{Nomenclature}

B

$\overline{\mathbf{b}}_{0}, \overline{\mathbf{b}}$

$\mathbf{C}$

$C_{i j}$

$\mathbb{C}$

$\mathbb{C}$

$c_{1}, c_{2}, c_{3}, c_{4}$

$\mathbf{E}$

$\mathbf{F}$

I

$I_{1}, I_{2}, I_{3}$

$J$

O

(1)

$\mathbf{P}$

$P_{j i}$

$p$

$\mathbf{S}$

$\overline{\mathbf{t}}_{0}, \overline{\mathbf{t}}$

$\overline{\mathbf{u}}_{0}, \overline{\mathbf{u}}$

$\mathbf{u}, \delta \mathbf{u}, \Delta \mathbf{u}$

$X_{1}, X_{2}, X_{3}$

$x_{1}, x_{2}, x_{3}$

$\mathbf{X}, \mathbf{Y}$

Subscripts

et

$i, j$

ps

t, $\mathrm{u}$ left Cauchy-Green tensor

specified body force vectors in reference and current configurations

right Cauchy-Green tensor

right Cauchy-Green tensor in indicial notation

fourth-order elasticity tensor in reference configuration

fourth-order elasticity tensor in current configuration

constitutive constants

Green-Lagrange strain tensor

deformation gradient tensor

second-order unit tensor

invariants of the right Cauchy-Green tensor

Jacobian or volume ratio

second-order null tensor

fourth-order null tensor

first Piola-Kirchhoff stress tensor or nominal stress tensor

nominal stress tensor in indicial notation

pressure or mean stress

second Piola-Kirchhoff stress tensor in reference configuration

specified traction vectors in reference and current configurations

specified displacement vectors in reference and current configurations

actual, virtual, and incremental displacement fields

Cartesian coordinates of a particle in reference configuration

Cartesian coordinates of a particle in current configuration

two different arbitrary second-order tensors

equibiaxial tension mode

index for three orthogonal directions

pure shear mode

traction and displacement 
ut

$0,1,2,3$

Greek Symbols

$\Gamma_{0}, \Gamma$

$\Delta_{1}, \Delta_{2}, \cdots, \Delta_{8}$

$\delta_{1}, \delta_{2}, \cdots, \delta_{8}$

$\delta_{i j}$

$\boldsymbol{\epsilon}$

$\kappa, \lambda, \nu$

$\sigma$

$\tau$

$\Psi$

$\Psi_{\mathrm{o}}$

$\Omega_{0}, \Omega$

Abbreviations

CSE

GSF

LLSQ

NR

SBR

TED uniaxial tension mode

reference configuration, length, width, and thickness directions

boundary in reference and current configurations

parameters for elasticity tensors in reference configuration

parameters for elasticity tensors in current configuration

Kronecker delta

Euler-Almansi strain tensor

shear stretch, normal stretch, Poisson index

Cauchy stress tensor in current configuration

Kirchhoff stress tensor in current configuration

general isotropic continuum stored energy functional

old isotropic continuum stored energy functional

domain in reference and current configurations

continuum stored energy

general stress-free

linear least squares

natural rubber

styrene-butadiene rubber

trial-and-error-on-digit

\section{Introduction}

All materials under loadings are compressible to a certain degree. Compressible mechanical behavior is related to varying stiffness combinations due to changes of distances, areas, and volumes in the fundamental metric of differential geometry. Without exception, rubber-like materials are nearly incompressible or hardly compressible at best.

Generally, perfect incompressibility is assumed for rubber-like materials, making analytical solutions of problems with simple geometry easier, but numerical finite element implementations more difficult [1]. As such, nearly incompressible finite element formulations have been developed. Difficulties in displacementbased finite element formulations, however, include ill-conditioning of the stiffness matrix, spurious or incorrect pressures, and element locking [2]. To overcome such difficulties, the mixed displacement/pressurebased finite element formulations have been developed. Nevertheless, mixed finite element formulations are not stable for all displacement and pressure fields and the deformation gradient is decomposed into isochoric and volumetric parts.

The multiplicative decomposition of the deformation gradient tensor into isochoric and volumetric parts in finite deformations was introduced by Richter [3], further studied by Flory [4], Lu and Pister [5], Sansour [6], and extensively applied by Lubliner [7], Simo and Taylor [8], Ogden [9], and many others. With the multiplicative decomposition of the deformation gradient, a stored-energy functional is additively decoupled into isochoric and volumetric parts. Subsequently, stress and elasticity tensors are also additively decoupled into isochoric and volumetric parts. The detailed derivations for the decoupled stress and elasticity tensors are documented by Holzapfel [10]. In commercial packages of finite element methods, ABAQUS, ANSYS, MARC, and NASTRAN offer Mooney-Rivilin model, Yeoh model, Ogden model, and other models. Some of the commercial packages also allow users to implement their own models in standard subroutines. Decoupled implementations, however, are not straightforward due to the complicated procedures in deriving stress and elasticity tensors. The decoupled stress and elasticity tensors for some models have been derived by Nicholson [11], Weiss, Maker, and Govindjee [12], Itskov [13], Suchocki [14], and Cheng and Zhang [15].

Accurate constitutive models will include compressibility [16]. The isochoric based compressible constitutive models for isotropic hyperelastic materials have been developed by Blatz and Ko [17], Ogden [18], Fried and Johnson [19], Anand [20], Bischoff, Arruda, and Grosh [21], and many others. Experimental 
characterizations and constitutive modelings of volume changes for stretched rubbers have been reviewed by Le Cam [22].

Uniaxial tension tests of rubber-like materials, however, show that the decomposition of a stored energy functional into isochoric and volumetric parts does not hold even at small deformations by Penn [23]. The finite deformations predicted by models with the isochoric-volumetric split are found to be unphysical by Ehlers and Eipper [24]. The isotropic CSE functional with symmetries resolved by Lie group methods and differential geometry - inherently no isochoric-volumetric split for finite deformation-has been developed by Zhao [25]. The isotropic CSE functional reads

$$
\Psi_{\mathrm{o}}=c_{1} I_{1}+c_{2} \sqrt{I_{2}}+c_{3} \frac{I_{1}^{4}}{I_{3}}
$$

where the three invariants of right Cauchy-Green tensor $\mathbf{C}, I_{1}, I_{2}$, and $I_{3}$, are defined by

$$
I_{1}=\mathbf{C}: \mathbf{I}=\operatorname{tr} \mathbf{C}, \quad I_{2}=\frac{1}{2}\left[(\operatorname{tr} \mathbf{C})^{2}-\operatorname{tr} \mathbf{C}^{2}\right], \quad I_{3}=\operatorname{det} \mathbf{C},
$$

and $\mathbf{I}$ is the second-order unit tensor and the matrix operators, ':', 'tr', and 'det', denote double contraction, trace, and determinant operations, respectively. The three constitutive constants, $c_{1}, c_{2}$ and $c_{3}$ are generally determined by experimental tests for volume changeability and used for modeling, predicting, and implementing finite deformations of isotropic hyperelastic materials.

The first-order derivatives of invariants are given by

$$
\frac{\partial I_{1}}{\partial \mathbf{C}}=\mathbf{I}, \quad \frac{\partial I_{2}}{\partial \mathbf{C}}=I_{1} \mathbf{I}-\mathbf{C}, \quad \frac{\partial I_{3}}{\partial \mathbf{C}}=I_{3} \mathbf{C}^{-1} .
$$

The second-order derivatives of invariants are given by

$$
\frac{\partial^{2} I_{1}}{\partial \mathbf{C}^{2}}=\mathbb{O}, \quad \frac{\partial^{2} I_{2}}{\partial \mathbf{C}^{2}}=\mathbf{I} \otimes \mathbf{I}-\mathbf{I} \odot \mathbf{I}, \quad \frac{\partial^{2} I_{3}}{\partial \mathbf{C}^{2}}=I_{3}\left(\mathbf{C}^{-1} \otimes \mathbf{C}^{-1}-\mathbf{C}^{-1} \odot \mathbf{C}^{-1}\right),
$$

where $\mathbb{O}$ is the fourth-order null tensor. The derivatives of second-order symmetric tensors are defined as

$$
\frac{\partial \mathbf{C}}{\partial \mathbf{C}}=\mathbf{I} \odot \mathbf{I}=(\mathbf{I} \odot \mathbf{I})_{i j k l}=\frac{1}{2}\left(\delta_{i k} \delta_{j l}+\delta_{i l} \delta_{j k}\right),
$$

in which $\delta_{i k}$, for example, is the Kronecker delta and

$$
\frac{\partial \mathbf{C}^{-1}}{\partial \mathbf{C}}=-\mathbf{C}^{-1} \odot \mathbf{C}^{-1}=-\left(\mathbf{C}^{-1} \odot \mathbf{C}^{-1}\right)_{i j k l}=-\frac{1}{2}\left(C_{i k}^{-1} C_{j l}^{-1}+C_{i l}^{-1} C_{j k}^{-1}\right) .
$$

For general CSE constitutive models of uniaxial tension tests, lateral contractions, $\lambda_{2}$ or $\lambda_{3}$, can be related to axial stretch, $\lambda_{1}$, with the Poisson or power function [17],

$$
\lambda_{2}=\lambda_{1}^{-\nu},
$$

where $\nu$ is the Poisson index. For the stress-free condition of $P_{3}=0$, a more general kinematic relation among three orthogonal stretches can be readily established based on the generalized Hooke's law

$$
\lambda_{3}=\left(\lambda_{1} \lambda_{2}\right)^{-\frac{\nu}{1-\nu}}
$$

In order to solve practical boundary-value problems associated with isotropic hyperelastic materials, nonlinear finite element procedures of total and updated Lagrangian formulations respective to corresponding reference and current configurations are desired.

For incremental total Lagrangian formulations, the linearization of the principle of virtual work with respect to displacements reads

$$
\begin{aligned}
\int_{\Omega_{0}}\left[\delta \mathbf{u}^{\mathrm{T}}\left(\partial_{\mathbf{u}} \mathbf{E}\right)^{\mathrm{T}}: \mathbb{C}: \partial_{\mathbf{u}} \mathbf{E}+\delta \mathbf{u}^{\mathrm{T}}\left(\partial_{\mathbf{u u}}^{2} \mathbf{E}\right)^{\mathrm{T}}: \mathbf{S}\right] \mathrm{d} \Omega_{0} \Delta \mathbf{u}= & \int_{\Omega_{0}} \delta \mathbf{u}^{\mathrm{T}} \cdot \overline{\mathbf{b}}_{0} \mathrm{~d} \Omega_{0}+\int_{\Gamma_{0 \mathrm{t}}} \delta \mathbf{u}^{\mathrm{T}} \cdot \overline{\mathbf{t}}_{0} \mathrm{~d} \Gamma_{0} \\
& -\int_{\Omega_{0}} \delta \mathbf{u}^{\mathrm{T}}\left(\partial_{\mathbf{u}} \mathbf{E}\right)^{\mathrm{T}}: \mathbf{S} \mathrm{d} \Omega_{0}
\end{aligned}
$$


where the superscript ${ }^{\mathrm{T}}$ is a transpose operator, $\mathbf{E}=(\mathbf{C}-\mathbf{I}) / 2$ is the Green-Lagrange strain tensor, $\mathbf{S}$ is the second Piola-Kirchhoff stress tensor, $\mathbb{C}$ is the fourth-order elasticity tensor in reference configuration, and the continuum body in domain $\Omega_{0}$ is bounded by boundary $\Gamma_{0}$. The boundary is partitioned into disjoint parts of $\Gamma_{0}=\Gamma_{0 \mathrm{t}} \cup \Gamma_{0 \mathrm{u}}$ with $\Gamma_{0 \mathrm{t}} \cap \Gamma_{0 \mathrm{u}}=\emptyset$. For domain conditions, $\overline{\mathbf{b}}_{0}$ is the specified body force in domain $\Omega_{0}$. For boundary conditions, $\overline{\mathbf{t}}_{0}$ is the specified traction on boundary $\Gamma_{0 \mathrm{t}}$ and $\overline{\mathbf{u}}_{0}$ is the specified displacement on boundary $\Gamma_{0 u}$.

For incremental updated Lagrangian formulations, the linearization of the principle of virtual work with respect to displacements is

$$
\begin{aligned}
\int_{\Omega}\left[\delta \mathbf{u}^{\mathrm{T}}\left(\partial_{\mathbf{u}} \boldsymbol{\epsilon}\right)^{\mathrm{T}}: \mathbb{C}: \partial_{\mathbf{u}} \boldsymbol{\epsilon}+\delta \mathbf{u}^{\mathrm{T}}\left(\partial_{\mathbf{u u}}^{2} \boldsymbol{\epsilon}\right)^{\mathrm{T}}: \boldsymbol{\sigma}\right] \mathrm{d} \Omega \Delta \mathbf{u}= & \int_{\Omega} \delta \mathbf{u}^{\mathrm{T}} \cdot \overline{\mathbf{b}} \mathrm{d} \Omega+\int_{\Gamma_{\mathrm{t}}} \delta \mathbf{u}^{\mathrm{T}} \cdot \overline{\mathbf{t}} \mathrm{d} \Gamma \\
& -\int_{\Omega} \delta \mathbf{u}^{\mathrm{T}}\left(\partial_{\mathbf{u}} \boldsymbol{\epsilon}\right)^{\mathrm{T}}: \boldsymbol{\sigma} \mathrm{d} \Omega
\end{aligned}
$$

where $\boldsymbol{\epsilon}=\left(\mathbf{I}-\mathbf{B}^{-1}\right) / 2$ is the Euler-Almansi strain tensor, $\mathbf{B}$ is the left Cauchy-Green tensor, $\boldsymbol{\sigma}$ is the Cauchy stress tensor, $\mathbb{C}$ is the fourth-order elasticity tensor in current configuration, and the continuum body in domain $\Omega$ is bounded by a boundary $\Gamma$. The boundary is partitioned into disjoint parts of $\Gamma=\Gamma_{\mathrm{t}} \cup \Gamma_{\mathrm{u}}$ with $\Gamma_{\mathrm{t}} \cap \Gamma_{\mathrm{u}}=\emptyset$. For domain conditions, $\overline{\mathrm{b}}$ is the specified body force in domain $\Omega$. For boundary conditions, $\overline{\mathbf{t}}$ is the specified traction on boundary $\Gamma_{\mathrm{t}}$ and $\overline{\mathbf{u}}$ is the specified displacement on boundary $\Gamma_{\mathrm{u}}$.

The finite element unknown variables, $\mathbf{u}, \delta \mathbf{u}$, and $\Delta \mathbf{u}$ are actual, virtual, and incremental displacement fields. The virtual displacement field $\delta \mathbf{u}$ as an arbitrary vector is essentially removed from all terms of (9) and (10), respectively. Specified displacements $\overline{\mathbf{u}}_{0}$ and $\overline{\mathbf{u}}$ are enforced in solution procedures albeit not shown. Fully detailed finite element implementations can be found in the books [26,27,28], among others.

In mixed finite element implementations with the isochoric-volumetric split, isochoric based constitutive models are usually adopted. The pressure--volume relation, with extra degrees of freedom, is needed to enforce the desired degree of compressibility. However, it is daunting to establish a physically realistic pressure-volume relation, let alone rigorously examining the validity of the isochoric-volumetric split. To overcome the major difficulty of establishing a physically realistic pressure-volume relation, the CSE functional as a non-isochoric based stored energy functional can be applied. The isotropic CSE functional (1), however, has been applied to constitutively model mechanical behaviors of rubbers with the Mullins effect removed. For a wide range of applications including the Mullins effect, the CSE functional will be generalized.

The major objectives, therefore, are to generalize the isotropic CSE functional, to constitutively model and implement compressible finite deformation without the isochoric-volumetric split, to derive the coupled stress and elasticity tensors in reference and current configurations, to examine the validity of the isochoric--volumetric split, and to characterize kinematic relation in compressibility tests.

\section{Compressible Isotropic CSE Models and Implementations}

\subsection{General Isotropic CSE Functional}

For wider and better modeling, predicting, and implementing, the isotropic CSE functional (1), similar to the anisotropic CSE functional developed by Zhao [29], can be generalized from the constant power of $c_{4}=1$ to the variable power of $c_{4}$ in the $c_{3}$ term as

$$
\Psi=c_{1} I_{1}+c_{2} \sqrt{I_{2}}+c_{3} \frac{I_{1}^{3 c_{4}+1}}{I_{3}^{c_{4}}},
$$

where the four constitutive constants, $c_{1}, c_{2}, c_{3}$, and $c_{4}$, will then be determined by experimental tests.

\subsection{General Isotropic CSE Constitutive Models}

Nominal stress and stretch results are preferably calculated from force and extension measurements with original effective sample dimensions recorded in experimental tests. The first Piola-Kirchhoff stress tensor 
or the nominal stress tensor, $\mathbf{P}$, is related to the second Piola-Kirchhoff stress tensor, $\mathbf{S}$, through the deformation gradient tensor, $\mathbf{F}$, by $\mathbf{P}=\mathbf{F S}$ or equivalently by

$$
\mathbf{P}^{\mathrm{T}}=\frac{\partial \Psi}{\partial \mathbf{F}}=2\left(\frac{\partial \Psi}{\partial I_{1}} \frac{\partial I_{1}}{\partial \mathbf{C}}+\frac{\partial \Psi}{\partial I_{2}} \frac{\partial I_{2}}{\partial \mathbf{C}}+\frac{\partial \Psi}{\partial I_{3}} \frac{\partial I_{3}}{\partial \mathbf{C}}\right)\left(\frac{1}{2} \frac{\partial \mathbf{C}}{\partial \mathbf{F}}\right)=\mathbf{S F}^{\mathrm{T}}
$$

where the nominal stress in indicial notation reads

$$
P_{j i}=\frac{\partial \Psi}{\partial I_{1}} \frac{\partial I_{1}}{\partial \lambda_{i j}}+\frac{\partial \Psi}{\partial I_{2}} \frac{\partial I_{2}}{\partial \lambda_{i j}}+\frac{\partial \Psi}{\partial I_{3}} \frac{\partial I_{3}}{\partial \lambda_{i j}}, \quad(i, j=1,2,3)
$$

The first-order derivatives of the general isotropic CSE functional, with all three invariants generally treated as variables, are

$$
\frac{\partial \Psi}{\partial I_{1}}=c_{1}+c_{3}\left(3 c_{4}+1\right) \frac{I_{1}^{3 c_{4}}}{I_{3}^{c_{4}}}, \quad \frac{\partial \Psi}{\partial I_{2}}=\frac{c_{2}}{2 \sqrt{I_{2}}}, \quad \frac{\partial \Psi}{\partial I_{3}}=-c_{3} c_{4} \frac{I_{1}^{3 c_{4}+1}}{I_{3}^{c_{4}+1}} .
$$

The second-order derivatives of the general isotropic CSE functional, with all three invariants generally treated as variables, are

$$
\begin{aligned}
& \frac{\partial^{2} \Psi}{\partial I_{1}^{2}}=3 c_{3} c_{4}\left(3 c_{4}+1\right) \frac{I_{1}^{3 c_{4}-1}}{I_{3}^{c_{4}}}, \quad \frac{\partial^{2} \Psi}{\partial I_{2}^{2}}=-\frac{c_{2}}{4 \sqrt{I_{2}^{3}}}, \quad \frac{\partial^{2} \Psi}{\partial I_{3}^{2}}=c_{3} c_{4}\left(c_{4}+1\right) \frac{I_{1}^{3 c_{4}+1}}{I_{3}^{c_{4}+2}}, \\
& \frac{\partial^{2} \Psi}{\partial I_{1} \partial I_{2}}=\frac{\partial^{2} \Psi}{\partial I_{2} \partial I_{3}}=0, \quad \frac{\partial^{2} \Psi}{\partial I_{1} \partial I_{3}}=-c_{3} c_{4}\left(3 c_{4}+1\right) \frac{I_{1}^{3 c_{4}}}{I_{3}^{c_{4}+1}} .
\end{aligned}
$$

Substituting (14), $I_{1}=I_{2}=3, I_{3}=1$, and derivatives of invariants with respect to normal stretches into (13) yields the general stress-free (GSF) condition in reference configuration

$$
c_{1}+\frac{c_{2}}{\sqrt{3}}+c_{3} 27^{c_{4}}=0 .
$$

Experimental tests of isotropic hyperelastic materials have effectively been conducted by uniaxial tension, pure shear, and equibiaxial tension tests. Thus, the general CSE constitutive models in three deformation modes will be derived based on the equations (2) and (13) through (17).

Uniaxial Tension Mode. The deformation of uniaxial tension can be generally modeled as

$$
x_{1}=\lambda_{1} X_{1}, \quad x_{2}=\lambda_{2} X_{2}, \quad x_{3}=\lambda_{3} X_{3} .
$$

For isotropic hyperelastic materials, we have $\lambda_{2}=\lambda_{1}^{-\nu}$ and $\lambda_{3}=\lambda_{1}^{-\nu}$ due to Poisson function (7), yielding $\lambda_{2}=\lambda_{3}$. The related tensors and invariants for the uniaxial tension mode in (18) are $\mathbf{F}=\operatorname{diag}\left[\lambda_{1}, \lambda_{2}, \lambda_{2}\right]$, $\mathbf{C}=\operatorname{diag}\left[\lambda_{1}^{2}, \lambda_{2}^{2}, \lambda_{2}^{2}\right], I_{1}=\lambda_{1}^{2}+2 \lambda_{2}^{2}, I_{2}=2 \lambda_{1}^{2} \lambda_{2}^{2}+\lambda_{2}^{4}, I_{3}=\lambda_{1}^{2} \lambda_{2}^{4}$, and their derivatives are

$$
\frac{\partial I_{1}}{\partial \lambda_{1}}=2 \lambda_{1}, \quad \frac{\partial I_{2}}{\partial \lambda_{1}}=4 \lambda_{1} \lambda_{2}^{2}, \quad \frac{\partial I_{3}}{\partial \lambda_{1}}=2 \lambda_{1} \lambda_{2}^{4}
$$

Substituting the derivatives (14), invariants, and derivatives of invariants (19) into (13) yields,

$$
P_{u t 1}=2 c_{1} \lambda_{1}+2 c_{2} \frac{\lambda_{1} \lambda_{2}^{2}}{\sqrt{2 \lambda_{1}^{2} \lambda_{2}^{2}+\lambda_{2}^{4}}}+2 c_{3} \frac{\left(\lambda_{1}^{2}+2 \lambda_{2}^{2}\right)^{3 c_{4}}}{\left(\lambda_{1}^{2} \lambda_{2}^{4}\right)^{c_{4}}}\left[\left(2 c_{4}+1\right) \lambda_{1}-2 c_{4} \frac{\lambda_{2}^{2}}{\lambda_{1}}\right],
$$

and the kinematic relation in uniaxial tension mode is given by the equation (7).

Pure Shear Mode. Pure shear tests have been conducted to validate the predictability of a model. In the pure shear mode, the lateral contraction is fixed with $\lambda_{2}=1$. Thus, the nominal stress is

$$
P_{p s 1}=2 c_{1} \lambda_{1}+c_{2} \frac{\lambda_{1}\left(1+\lambda_{3}^{2}\right)}{\sqrt{\lambda_{1}^{2}+\lambda_{3}^{2}+\lambda_{1}^{2} \lambda_{3}^{2}}}+2 c_{3} \frac{\left(\lambda_{1}^{2}+\lambda_{3}^{2}+1\right)^{3 c_{4}}}{\left(\lambda_{1}^{2} \lambda_{3}^{2}\right)^{c_{4}}}\left[\left(2 c_{4}+1\right) \lambda_{1}-c_{4} \frac{1+\lambda_{3}^{2}}{\lambda_{1}}\right],
$$

where thickness reduction, $\lambda_{3}$, can be obtained by substituting $\lambda_{2}=1$ into (8)

$$
\lambda_{3}=\lambda_{1}^{-\frac{\nu}{1-\nu}} .
$$


Equibiaxial Tension Mode. Equibiaxial tension tests have also been performed to validate the predictability of a model. In the equibiaxial tension mode, the in-plane stretches, $\lambda_{1}=\lambda_{2}$, are equally maintained or applied. Thus, the nominal stress is

$$
P_{e t 1}=2 c_{1} \lambda_{1}+c_{2} \frac{\left(\lambda_{1}^{2}+\lambda_{3}^{2}\right)}{\sqrt{\lambda_{1}^{2}+2 \lambda_{3}^{2}}}+2 c_{3} \frac{\left(2 \lambda_{1}^{2}+\lambda_{3}^{2}\right)^{3 c_{4}}}{\left(\lambda_{1}^{4} \lambda_{3}^{2}\right)^{c_{4}}}\left[\left(c_{4}+1\right) \lambda_{1}-c_{4} \frac{\lambda_{3}^{2}}{\lambda_{1}}\right],
$$

where thickness reduction, $\lambda_{3}$, can be worked out by substituting $\lambda_{2}=\lambda_{1}$ into (8)

$$
\lambda_{3}=\lambda_{1}^{-\frac{2 \nu}{1-\nu}}
$$

Equations (7), (22), and (24) will be used to model lateral contraction in uniaxial tension mode, thickness reduction in pure shear and equibiaxial tension modes, respectively.

\subsection{Stress Tensors in Reference and Current Configurations}

For the general CSE functional defined in (11), the second Piola-Kirchhoff stress tensor, S, in reference configuration is worked out as

$$
\mathbf{S}=\left[2 c_{1}+c_{2} \frac{I_{1}}{\sqrt{I_{2}}}+2 c_{3}\left(3 c_{4}+1\right) \frac{I_{1}^{3 c_{4}}}{I_{3}^{c_{4}}}\right] \mathbf{I}-\frac{c_{2}}{\sqrt{I_{2}}} \mathbf{C}-2 c_{3} c_{4} \frac{I_{1}^{3 c_{4}+1}}{I_{3}^{c_{4}}} \mathbf{C}^{-1},
$$

and the Cauchy stress tensor, $\boldsymbol{\sigma}$, in current configuration can be converted by the push-forward operation of (25) and readily obtained as

$$
\boldsymbol{\sigma}=\frac{1}{J} \mathbf{F S F}^{\mathrm{T}}=\frac{1}{J}\left\{\left[2 c_{1}+c_{2} \frac{I_{1}}{\sqrt{I_{2}}}+2 c_{3}\left(3 c_{4}+1\right) \frac{I_{1}^{3 c_{4}}}{I_{3}^{c_{4}}}\right] \mathbf{B}-\frac{c_{2}}{\sqrt{I_{2}}} \mathbf{B}^{2}-2 c_{3} c_{4} \frac{I_{1}^{3 c_{4}+1}}{I_{3}^{c_{4}}} \mathbf{I}\right\},
$$

where $J=\sqrt{I_{3}}$ is the Jacobian or volume ratio.

\subsection{Elasticity Tensors in Reference and Current Configurations}

For finite deformations of isotropic hyperelastic materials, the generally coupled fourth-order elasticity tensor in reference configuration reads

$$
\mathbb{C}=4 \sum_{i=1}^{3}\left[\sum_{j=1}^{3} \frac{\partial^{2} \Psi}{\partial I_{i} \partial I_{j}}\left(\frac{\partial I_{i}}{\partial \mathbf{C}} \otimes \frac{\partial I_{j}}{\partial \mathbf{C}}\right)+\frac{\partial \Psi}{\partial I_{i}} \frac{\partial^{2} I_{i}}{\partial \mathbf{C}^{2}}\right]
$$

Substituting the first-order and second-order derivatives of invariants (3) and (4), simplifying, and rearranging produces

$$
\begin{aligned}
\mathbb{C}= & \Delta_{1} \mathbf{I} \otimes \mathbf{I}+\Delta_{2}(\mathbf{I} \otimes \mathbf{C}+\mathbf{C} \otimes \mathbf{I})+\Delta_{3}\left(\mathbf{I} \otimes \mathbf{C}^{-1}+\mathbf{C}^{-1} \otimes \mathbf{I}\right)+\Delta_{4} \mathbf{C} \otimes \mathbf{C}+ \\
& \Delta_{5}\left(\mathbf{C} \otimes \mathbf{C}^{-1}+\mathbf{C}^{-1} \otimes \mathbf{C}\right)+\Delta_{6} \mathbf{C}^{-1} \otimes \mathbf{C}^{-1}+\Delta_{7} \mathbf{C}^{-1} \odot \mathbf{C}^{-1}+\Delta_{8} \mathbf{I} \odot \mathbf{I},
\end{aligned}
$$

where the eight parameters $\Delta_{1}, \Delta_{2}, \cdots, \Delta_{8}$ for a stored energy functional are defined by

$$
\begin{aligned}
& \Delta_{1}=4\left(\frac{\partial^{2} \Psi}{\partial I_{1}^{2}}+2 I_{1} \frac{\partial^{2} \Psi}{\partial I_{1} \partial I_{2}}+I_{1}^{2} \frac{\partial^{2} \Psi}{\partial I_{2}^{2}}+\frac{\partial \Psi}{\partial I_{2}}\right), \quad \Delta_{2}=-4\left(\frac{\partial^{2} \Psi}{\partial I_{1} \partial I_{2}}+I_{1} \frac{\partial^{2} \Psi}{\partial I_{2}^{2}}\right), \\
& \Delta_{3}=4\left(I_{3} \frac{\partial^{2} \Psi}{\partial I_{3} \partial I_{1}}+I_{3} I_{1} \frac{\partial^{2} \Psi}{\partial I_{2} \partial I_{3}}\right), \quad \Delta_{4}=4 \frac{\partial^{2} \Psi}{\partial I_{2}^{2}}, \quad \Delta_{5}=-4 I_{3} \frac{\partial^{2} \Psi}{\partial I_{2} \partial I_{3}}, \\
& \Delta_{6}=4\left(I_{3}^{2} \frac{\partial^{2} \Psi}{\partial I_{3}^{2}}+I_{3} \frac{\partial \Psi}{\partial I_{3}}\right), \quad \Delta_{7}=-4 I_{3} \frac{\partial \Psi}{\partial I_{3}}, \quad \Delta_{8}=-4 \frac{\partial \Psi}{\partial I_{2}} .
\end{aligned}
$$


For the general CSE functional, the eight parameters $\Delta_{1}, \Delta_{2}, \cdots, \Delta_{8}$, using (15) and (16), are more specifically given by

$$
\begin{aligned}
& \Delta_{1}=\frac{2 c_{2}}{\sqrt{I_{2}}}-\frac{c_{2} I_{1}^{2}}{\sqrt{I_{2}^{3}}}+12 c_{3} c_{4}\left(3 c_{4}+1\right) \frac{I_{1}^{3 c_{4}-1}}{I_{3}^{c_{4}}}, \quad \Delta_{2}=\frac{c_{2} I_{1}}{\sqrt{I_{2}^{3}}}, \quad \Delta_{3}=-4 c_{3} c_{4}\left(3 c_{4}+1\right) \frac{I_{1}^{3 c_{4}}}{I_{3}^{c_{4}}}, \\
& \Delta_{4}=-\frac{c_{2}}{\sqrt{I_{2}^{3}}}, \quad \Delta_{5}=0, \quad \Delta_{6}=4 c_{3} c_{4}^{2} \frac{I_{1}^{3 c_{4}+1}}{I_{3}^{c_{4}}}, \quad \Delta_{7}=4 c_{3} c_{4} \frac{I_{1}^{3 c_{4}+1}}{I_{3}^{c_{4}}}, \quad \Delta_{8}=-\frac{2 c_{2}}{\sqrt{I_{2}}} .
\end{aligned}
$$

The elasticity tensor in current configuration can be converted from the elasticity tensor in reference configuration by the following push-forward operation of (28)

$$
\mathbb{C}=\frac{1}{J}(\mathbf{F} \odot \mathbf{F}): \mathbb{C}:\left(\mathbf{F}^{\mathrm{T}} \odot \mathbf{F}^{\mathrm{T}}\right) .
$$

For the push-forward operation in (34), two important properties have been derived

$$
(\mathbf{F} \odot \mathbf{F}):(\mathbf{X} \otimes \mathbf{Y}):\left(\mathbf{F}^{\mathrm{T}} \odot \mathbf{F}^{\mathrm{T}}\right)=\left[\mathbf{F}\left(\frac{\mathbf{X}+\mathbf{X}^{\mathrm{T}}}{2}\right) \mathbf{F}^{\mathrm{T}}\right] \otimes\left[\mathbf{F}\left(\frac{\mathbf{Y}+\mathbf{Y}^{\mathrm{T}}}{2}\right) \mathbf{F}^{\mathrm{T}}\right],
$$

and

$$
(\mathbf{F} \odot \mathbf{F}):(\mathbf{X} \odot \mathbf{Y}):\left(\mathbf{F}^{\mathrm{T}} \odot \mathbf{F}^{\mathrm{T}}\right)=\frac{1}{2}\left[\left(\mathbf{F} \mathbf{X} \mathbf{F}^{\mathrm{T}}\right) \odot\left(\mathbf{F} \mathbf{Y} \mathbf{F}^{\mathrm{T}}\right)+\left(\mathbf{F} \mathbf{Y} \mathbf{F}^{\mathrm{T}}\right) \odot\left(\mathbf{F} \mathbf{X} \mathbf{F}^{\mathrm{T}}\right)\right],
$$

where $\mathbf{X}$ and $\mathbf{Y}$ are two arbitrary second-order tensors. In the derivation of (35) and (36), useful fundamental properties of double contraction operations between fourth-order tensors by Kintzel and Başar [30] and Kintzel [31] have been utilized.

With the properties of double contraction operations between fourth-order tensors (35) and (36), the general coupled fourth-order elasticity tensor in current configuration for isotropic hyperelastic materials, using (34), can be converted from the fourth-order elasticity tensor in reference configuration (28) as

$$
\begin{aligned}
\mathbb{C}= & \delta_{1}(\mathbf{B} \otimes \mathbf{B})+\delta_{2}\left(\mathbf{B} \otimes \mathbf{B}^{2}+\mathbf{B}^{2} \otimes \mathbf{B}\right)+\delta_{3}(\mathbf{I} \otimes \mathbf{B}+\mathbf{B} \otimes \mathbf{I})+\delta_{4}\left(\mathbf{B}^{2} \otimes \mathbf{B}^{2}\right)+ \\
& \delta_{5}\left(\mathbf{I} \otimes \mathbf{B}^{2}+\mathbf{B}^{2} \otimes \mathbf{I}\right)+\delta_{6}(\mathbf{I} \otimes \mathbf{I})+\delta_{7}(\mathbf{I} \odot \mathbf{I})+\delta_{8}(\mathbf{B} \odot \mathbf{B}),
\end{aligned}
$$

where the eight parameters $\delta_{1}, \delta_{2}, \cdots, \delta_{8}$ for the general CSE functional are given by

$$
\begin{aligned}
& \delta_{1}=\frac{2 c_{2}}{\sqrt{I_{2}} J}-\frac{c_{2} I_{1}^{2}}{\sqrt{I_{2}^{3}} J}++12 c_{3} c_{4}\left(3 c_{4}+1\right) \frac{I_{1}^{3 c_{4}-1}}{I_{3}^{c_{4} J}}, \quad \delta_{2}=\frac{c_{2} I_{1}}{\sqrt{I_{2}^{3}} J}, \quad \delta_{3}=-4 c_{3} c_{4}\left(3 c_{4}+1\right) \frac{I_{1}^{3 c_{4}}}{I_{3}^{c_{4} J}}, \\
& \delta_{4}=-\frac{c_{2}}{\sqrt{I_{2}^{3}} J}, \quad \delta_{5}=0, \quad \delta_{6}=4 c_{3} c_{4}^{2} \frac{I_{1}^{3 c_{4}+1}}{I_{3}^{c_{4} J}}, \quad \delta_{7}=4 c_{3} c_{4} \frac{I_{1}^{3 c_{4}+1}}{I_{3}^{c_{4}} J}, \quad \delta_{8}=-\frac{2 c_{2}}{\sqrt{I_{2} J}} .
\end{aligned}
$$

The coupled fourth-order elasticity tensors in both reference and current configurations (28) and (37) have been derived for the general isotropic CSE functional.

\section{Applications of General CSE Models}

\subsection{Modeling for Natural Rubber in Multi-axial Tension Tests}

Uniaxial tension, pure shear, and equibiaxial tension tests of compressible natural rubber (NR) have been conducted by Storåkers [32]. A self-developed graphics digitizer with MATLAB has been used to read out experimental data in the three deformation modes. The nominal stress-stretch data and lateral contraction-axial stretch data for compressible NR in uniaxial tension mode have been selected to fit the general CSE model (20) with the GSF condition (17) and kinematic relation (7), respectively. With 


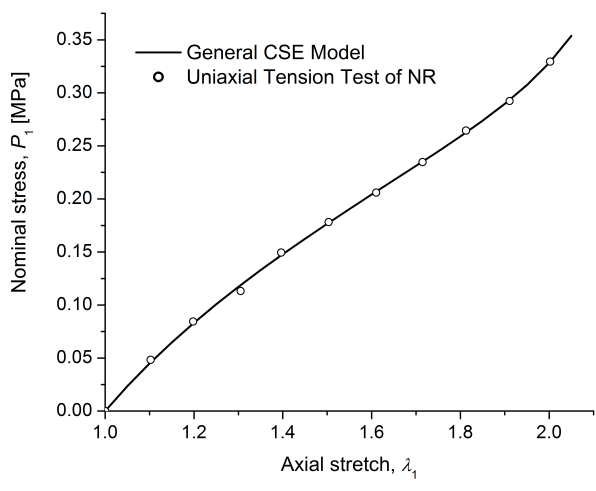

(a) stress-stretch relation

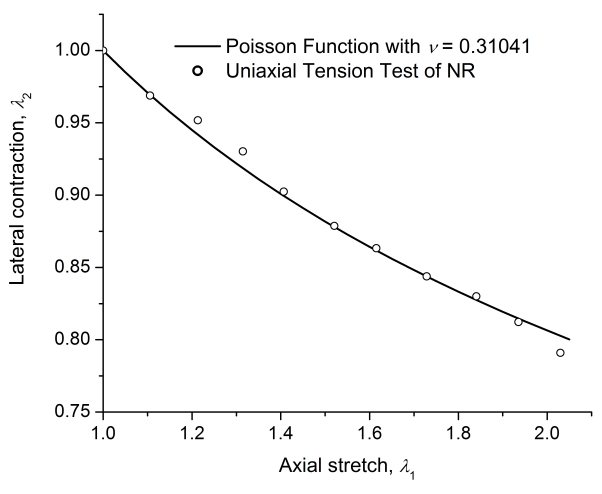

(b) contraction-stretch relation

Figure 1. Comparison between general CSE model and uniaxial tension test of NR.

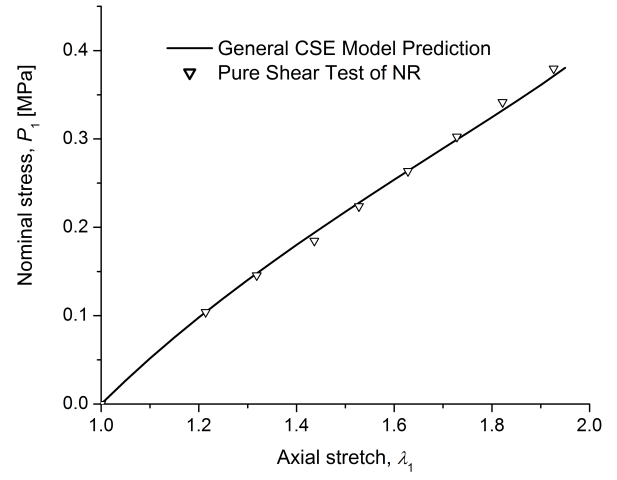

(a) stress-stretch relation

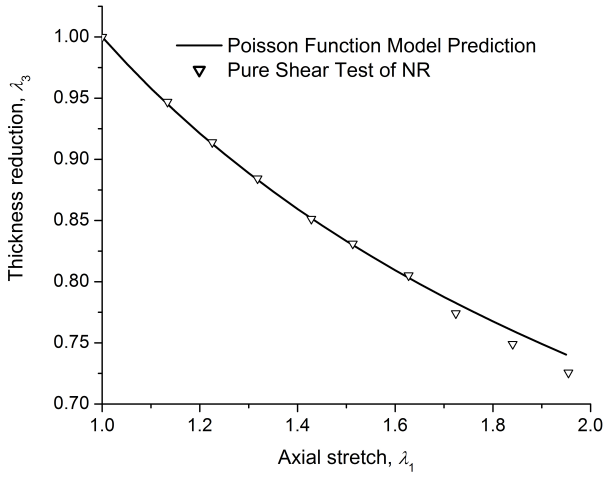

(b) reduction-stretch relation

Figure 2. Comparison between general CSE model prediction and pure shear test of NR.

curve-fitting, the Poisson index of $\nu=0.31041$ is determined. The constitutive constants in uniaxial tension mode have been solved by the trial-and-error-on-digit (TED) method and the linear least square (LLSQ) method combined [33]. The comparison between the general CSE model and the uniaxial tension test data, along with the lateral contraction-axial stretch curve, is shown in Figure 1.

The constitutive constants and $\nu=0.31041$ obtained in uniaxial tension mode have been submitted into (21) and (22) respectively to predict the compressible finite deformation in pure shear mode. The comparison between the general CSE model and the pure shear test data, along with the kinematic prediction, is shown in Figure 2.

The constitutive constants and $\nu=0.31041$ fitted in uniaxial tension mode have similarly been submitted into (23) and (24) respectively to predict the compressible finite deformation in equibiaxial tension mode. The comparison between the general CSE model and the equibiaxial tension test data, along with the corresponding kinematic prediction, is shown in Figure 3.

\subsection{Modeling of Styrene-Butadiene Rubber in Uniaxial Tension Tests}

Uniaxial tension tests of compressible styrene-butadiene rubber (SBR) filled with different volumepercentages of silica particles have been conducted by Starkova and Aniskevich [34]. At least three dumbbell shaped samples were tested at room temperature. A self-developed graphics digitizer with 


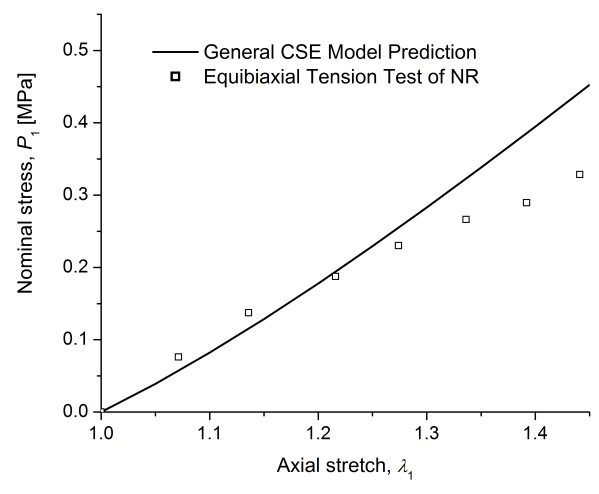

(a) stress-stretch relation

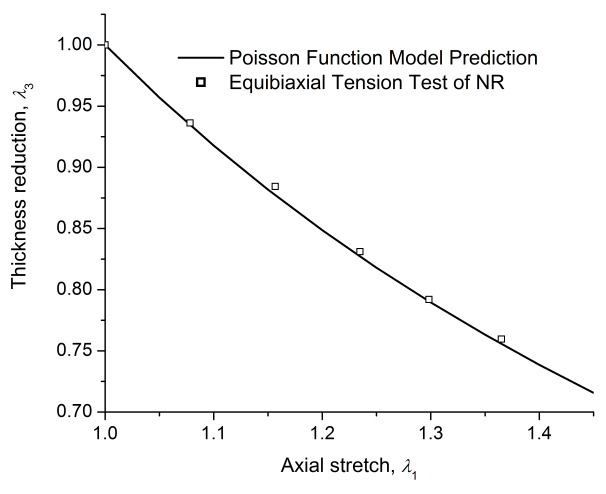

(b) reduction-stretch relation

Figure 3. Comparison between general CSE model prediction and equibiaxial tension test of NR.

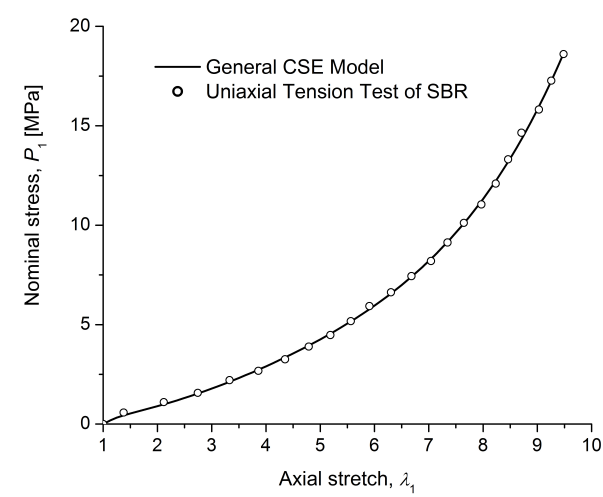

(a) stress-stretch relation

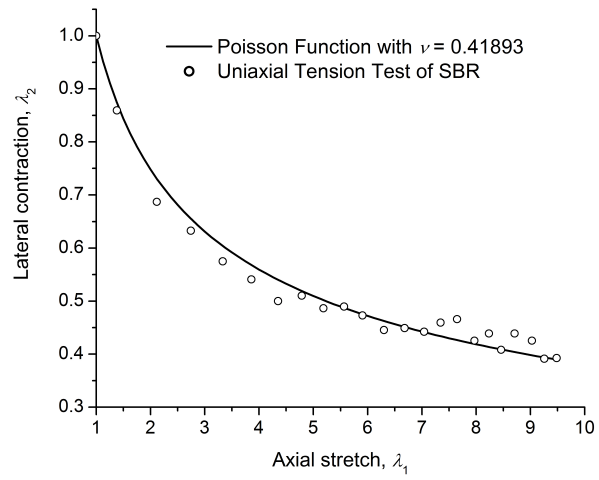

(b) contraction-stretch relation

Figure 4. Comparison between general CSE model and uniaxial tension test of SBR.

MATLAB has been used to read out experimental data of nominal stress $P_{1}$ and stretch $\lambda_{1}$ as well as those of axial stretch $\lambda_{1}$ and lateral contraction $\lambda_{2}$. The lateral contraction is assumed to relate axial stretch by the Poisson function (7). With curve-fitting, the Poisson index of compressible SBR is $\nu=0.41893$. Uniaxial tension test data for SBR filled with $14.11 \%$ of silica particles, exhibiting volume-expandable behaviors, have been selected to fit the general CSE model (20) with the GSF condition (17). The maximum ratio of current volume to original volume is 1.44018 at the maximum axial stretch of 9.48485, indicating that the sample volume increases by about $44 \%$ in the uniaxial tension test. The constitutive constants have been solved by the TED-LLSQ method. The comparison between the general CSE model and the experimental data, along with the lateral contraction-axial stretch curve, is shown in Figure 4.

\section{Discussion}

Constitutive modeling of isotropic hyperelastic materials in finite deformations requires good theoretical models and suitable experimental characterizations. As a common practice, theoretical models are fitted with experimental data tested in certain deformation modes and the fitted models predict finite deformations in unfitted and untested modes. The theoretical development of constitutive models, the optimal design of experimental tests, and their numerical implementations into finite element 
Table 1. Constitutive Constants of General CSE Models for Rubbers.

\begin{tabular}{lrrccc}
\hline Material & $c_{1}(\mathrm{MPa})$ & $c_{2}(\mathrm{MPa})$ & $c_{3}(\mathrm{MPa})$ & $c_{4}$ & $\nu$ \\
\hline NR & 0.17929 & 0.89776 & $5.37199 \times 10^{-14}$ & 5.15290 & 0.31041 \\
SBR & 0.71843 & 4.07522 & $8.82102 \times 10^{-06}$ & 0.77831 & 0.41893 \\
\hline
\end{tabular}

methods are crucial in real-world practical analyses and designs involving isotropic hyperelastic materials. Accurate constitutive models fit tested deformation modes, predict untested deformation modes, guide optimal designs of experiments, and even correct experimental errors while experimental tests determine constitutive constants of models and give feedbacks of models.

\subsection{Constitutive Modeling of Rubbers}

For general isotropic CSE models, the constitutive constants for the two different isotropic hyperelastic materials are listed in Table 1. The constitutive constants fitted from uniaxial tension tests of compressible NR shown in Figure 1 are used to predict finite deformations at pure shear mode shown in Figure 2 and at equibiaxial tension mode shown in Figure 3. As shown, the predictions are accurate for the pure shear mode at all stretches and overestimated the equibiaxial tension mode at small stretches but underestimated at relatively larger stretches. The overestimation is common for equibiaxial tension mode due to ignored shear stretch albeit only $1 \%$ of normal stretch in magnitude. The underestimation at relatively larger stretches for the equibiaxial tension mode might be the branch due to bifurcation issues [32]. For larger stretches of compressible SBR, the general CSE model and the Poisson function fit both uniaxial tension tests and the kinematic relation accurately as shown in Figure 4. For the studied compressible NR and SBR in uniaxial tension tests, the Poisson indexes are not even close to $\nu=0.5$ defined as an ideal incompressibility.

\subsection{Experimental Tests of Compressibility}

Uniaxial tension/compression, confined uniaxial compression, and hydrostatic compression or pure dilatation tests are usually used to characterize compressibility of isotropic hyperelastic materials [35]. In general, a stored energy functional cannot capture stretches without stress work done. Thus, the Poisson index relates a principal stretch with stress work done to other orthogonal principal stretches without stress work done in experimental tests. The uniaxial tension test is still one of the most accurate characterization methods. Unconfined uniaxial compression tests must be conducted with extra care in lubrication to maintain uniform deformations (without barreling). The confined uniaxial compression test becomes a confined volumetric test since the special relations of $\lambda_{2}=\lambda_{3}=1, \lambda_{1}=J$, and $P_{1}=p$ hold, in which $p$ is pressure. Intrinsic lateral deformation effects resulting from axial deformation, however, are suppressed for all materials with the Poisson index of $\nu=0.0$, forming one extreme. The other extreme is the perfect incompressibility with the Poisson index of $\nu=0.5$. From the tested isotropic hyperelastic materials, the Poisson indexes for finite deformations fall within the range of $0.0<\nu<0.5$. Thus, semi-confined uniaxial compression tests, rather than confined uniaxial compression tests, are slightly better at characterizing compressibility of isotropic hyperelastic materials. Hydrostatic compression or pure dilatation tests of compressibility, however, are not recommended.

For uniaxial tension and compression tests of isotropic hyperelastic materials, incompressibility is also used to calculate the deformed cross section and Cauchy stress. With the assumption of incompressibility, the Cauchy stress is overestimated for uniaxial tension tests with underestimated lateral contraction while the Cauchy stress is underestimated for uniaxial compression tests with overestimated lateral stretch. Thus, both axial and lateral stretches must actually be measured in the experimental tests $[34,36]$.

\subsection{The Isochoric-Volumetric Split}

The isochoric-volumetric split results in the additive decomposition of stored energy functionals, constructing decoupled formulations. Decoupled algorithms constitute the canonical procedures of finite deformation 
computations in commercial software packages for isotropic and anisotropic hyperelastic materials. For finite element implementations, the validity of the isochoric-volumetric split will be discussed based on stretch, stress, and elasticity measures.

Stretch Measures. Finite deformations are fundamentally measured through the deformation gradient tensor with three normal stretches, which provide distance change information, and six shear stretches, which provide angle change information. The isochoric and volumetric deformations are secondary definitions derived from the fundamental definitions. Intrinsic Poisson effects of hyperelastic materials are suppressed in volumetric deformation modes. Instead of the pressure-volume relation, the characterization of Poisson indexes is a key to model compressible finite deformations of isotropic hyperelastic materials.

The incompressible deformation is a special case of general deformations. When $I_{3}$ is treated as a variable, the general deformation includes incompressible deformations of $I_{3}=1$ and nearly incompressible deformations of $I_{3} \approx 1$. When $I_{3}$ is treated as a constant, however, part of deformation due to $I_{3}$ is eliminated by differentiations.

Stress Measures. Stresses are fundamentally evaluated by three normal stress components and three shear stress components due to symmetry in the second Piola-Kirchhoff stress tensor or the Cauchy stress tensor. The deviatoric stress and volumetric stress tensors are secondary definitions derived from the fundamental definitions. The second Piola-Kirchhoff stress tensor or the Cauchy stress tensor is generally given by

$$
\mathbf{S}=2\left[\left(\frac{\partial \Psi}{\partial I_{1}}+I_{1} \frac{\partial \Psi}{\partial I_{2}}\right) \mathbf{I}-\frac{\partial \Psi}{\partial I_{2}} \mathbf{C}+I_{3} \frac{\partial \Psi}{\partial I_{3}} \mathbf{C}^{-1}\right]
$$

or

$$
\boldsymbol{\sigma}=\frac{2}{J}\left[\left(\frac{\partial \Psi}{\partial I_{1}}+I_{1} \frac{\partial \Psi}{\partial I_{2}}\right) \mathbf{B}-\frac{\partial \Psi}{\partial I_{2}} \mathbf{B}^{2}+I_{3} \frac{\partial \Psi}{\partial I_{3}} \mathbf{I}\right]
$$

Neither is the partial derivative $\partial \Psi / \partial I_{1}$ necessarily only a function of $I_{1}$, nor is the partial derivative $\partial \Psi / \partial I_{3}$ only a function of $I_{3}$. Thus, the last term of (40) or (41) is not a pure hydrostatic stress term. Furthermore, the assumption of the isochoric-volumetric split is equivalent to assume that hydrostatic stress should only depend on volume change $[37,38]$. Hydrostatic stresses can generally be evaluated by taking one-third of the trace of (40) or (41), respectively

$$
\frac{1}{3} \operatorname{tr} \mathbf{S}=\frac{2}{3}\left(3 \frac{\partial \Psi}{\partial I_{1}}+2 I_{1} \frac{\partial \Psi}{\partial I_{2}}+I_{2} \frac{\partial \Psi}{\partial I_{3}}\right),
$$

or

$$
\frac{1}{3} \operatorname{tr} \boldsymbol{\sigma}=\frac{2}{3 J}\left(I_{1} \frac{\partial \Psi}{\partial I_{1}}+2 I_{2} \frac{\partial \Psi}{\partial I_{2}}+3 I_{3} \frac{\partial \Psi}{\partial I_{3}}\right)
$$

Hydrostatic stresses from both (42) and (43) are function of $I_{1}, I_{2}$, and $I_{3}$, indicating the assumption of hydrostatic stress only as a function of $I_{3}$ is not true. For the general isotropic CSE functional, the second Piola-Kirchhoff stress tensor and the Cauchy stress tensor are given in (25) and (26), respectively. The third and fifth terms in (25) or (26) contain $I_{3}^{c_{4}}$ coupled with $I_{1}^{3 c_{4}}$ and $I_{1}^{3 c_{4}+1}$, respectively. Thus, neither the second Piola-Kirchhoff stress tensor nor the Cauchy stress tensor can be decoupled into deviatoric stress tensor and hydrostatic stress tensor in the finite deformation domain. Furthermore, the stress is not just divided into two parts, deviatoric and hydrostatic, for finite elastic as well as plastic deformations. For the general isotropic CSE functional, stress is generally composed of three indispensable parts of contributions: distance-, area-, and volume-changes in the metric of differential geometry [25,39].

The partial differential equation of the general CSE functional can be recovered by rearranging (43) and using (11) and (14)

$$
\frac{J}{2} \operatorname{tr} \boldsymbol{\sigma}=\boldsymbol{\sigma}: \frac{\mathbf{I}}{2} J=I_{1} \frac{\partial \Psi}{\partial I_{1}}+2 I_{2} \frac{\partial \Psi}{\partial I_{2}}+3 I_{3} \frac{\partial \Psi}{\partial I_{3}}=\Psi
$$


where the general CSE functional $\Psi$ is indeed an invariant since the mean Cauchy stress is an invariant, making the CSE functional frame-indifferent. The general CSE functional as an invariant can be further demonstrated by the following equivalent stress work done in isothermal processes

$$
\mathbf{P}: \frac{\mathbf{F}}{2}=\mathbf{S}: \frac{\mathbf{C}}{2}=\boldsymbol{\tau}: \frac{\mathbf{I}}{2}=\boldsymbol{\sigma}: \frac{\mathbf{I}}{2} J,
$$

where $\boldsymbol{\tau}$ is the Kirchhoff stress tensor and the work-conjugacy is preserved.

Elasticity Measures. Furthermore, the parameters contained with $I_{3}$ of elasticity tensors are coupled with $I_{1}$. Thus, elasticity tensors cannot be additively decomposed into isochoric and volumetric parts.

For incremental finite element formulations of finite deformations, the Truesdell rate of stress should be used since it is a work-conjugate implementation. Large errors in finite element predictions could occur as a result of violating the requirement of work-conjugacy, especially for highly compressible materials [40,41]. In current incremental finite element implementations with the isochoric-volumetric split, ABAQUS uses the Jaumann stress rate, ANSYS adopts the Green-Naghdi stress rate, and NASTRAN selects the Truesdell stress rate formulations [42]. With the general CSE functional, the incremental finite element implementations of finite deformations with the Truesdell rate of stress, without the isochoric-volumetric split or incompressibility, has evidentially been even better for numerical properties in accuracy, efficiency, and stability.

\subsection{Summary}

The relevant features regarding to constitutive modelings and finite element implementations of general finite deformations for isotropic hyperelastic materials are emphasized and summarized as follows:

- The isotropic CSE functional (1) has been generalized from the constant power of $c_{4}=1$ to the variable power of $c_{4}$, establishing the general CSE functional (11) for both unfilled and filled rubbers;

- In finite element implementations without the isochoric-volumetric split, the coupled stress and elasticity tensors in reference and current configurations have been derived for the general isotropic CSE functional;

- The general CSE models have been applied to model and predict mechanical responses of compressible NR including kinematic relations in uniaxial tension, pure shear, and equibiaxial tension modes. Additionally, uniaxial tension tests of compressible SBR with much greater stretches have also successfully been modeled;

- In the uniaxial tension tests of compressible NR and SBR, the fitted Poisson indexes listed in Table 1 are not even close to $\nu=0.5$ defined as an idealized incompressibility;

- With the incompressibility condition, the Cauchy stress is overestimated for uniaxial tension tests with underestimated lateral contraction while the Cauchy stress is underestimated for uniaxial compression tests with overestimated lateral stretch;

- Mean stresses or pressures in terms of both the second Piola-Kirchhoff stress tensor (42) and the Cauchy stress tensor (43) are function of $I_{1}, I_{2}$, and $I_{3}$. Thus, the deformation of a material under pressure due to distance-, area-, and volume-changes are indispensable and inseparable. Therefore, it is daunting to distinguish what portion of applied pressure changes distances, what portion of applied pressure changes areas, and what portion of applied pressure changes volume either in experimental tests or in theoretical analyses;

- The assumption of the isochoric-volumetric split is equivalent to assume that hydrostatic stress should only depend on volume change $[37,38]$. The isochoric-volumetric split is invalid based on either theoretical analyses through stretch, stress, and elasticity measures in the current study or other experimental validations $[23,24]$;

- Instead of irrational pressure-volume relation, three orthogonal stretches, $\lambda_{1}, \lambda_{2}, \lambda_{3}$, for anisotropic soft biological tissues and two orthogonal stretches, $\lambda_{1}, \lambda_{2}=\lambda_{3}$, for isotropic hyperelastic materials should be measured simultaneously in uniaxial tension tests for compressibility. A principal stretch with stress work done, $\lambda_{1}$, should be related to other orthogonal principal stretches without stress work done, $\lambda_{2}$ and $\lambda_{3}$, through the Poisson index in uniaxial tension tests; 
- The general CSE functional is consistent in work-conjugate formulations through modeling, predicting, and implementing. Nevertheless, the equivalent work done used to establish the general CSE functional in isothermal processes by four different conjugate pairs commonly used in continuum mechanics is given in (45).

\section{Conclusions}

The general CSE functional (11) is applied to model and implement compressible finite deformations of isotropic hyperelastic materials without the isochoric-volumetric split. The related stress and elasticity tensors in reference and current configurations have been derived. The isochoric-volumetric split is invalid based on either the theoretical analyses or the experimental validations. Kinematic relations by Poisson function as an example, rather than irrational pressure--volume relation, should be characterized in uniaxial tension tests of compressible rubbers. The Poisson index should relate a principal stretch with stress work done to other orthogonal principal stretches without stress work done.

The general CSE constitutive models, along with the Poisson function, have been applied to curve-fit the uniaxial tension test of compressible NR and to predict corresponding pure shear and equibiaxial tension behaviors. Additionally, uniaxial tension tests of compressible SBR bars with much greater stretches are also successfully modeled.

The general CSE functional will be extensively applied to a wide range of mechanical responses for both unfilled and filled rubber-like materials.

Acknowledgments. The author is immensely grateful to Jianming and Jiesi Zhao for their support, encouragement, and assistance.

\section{References}

1. I. Fried, "Reflections on the computational approximation of elastic incompressibility," Computers \& Structures, vol. 17 , no. 2, pp. 161-168, 1983.

2. T. Sussman and K.-J. Bathe, "A finite element formulation for nonlinear incompressible elastic and inelastic analysis," Computers \& Structures, vol. 26, no. 1-2, pp. 357-409, 1987.

3. H. Richter, "Das isotrope elastizitätsgesetz," Zeitschrift für angewandte Mathematik und Mechanik, vol. 28, no. 7-8, pp. 205-209, 1948.

4. P. J. Flory, "Thermodynamic relations for high elastic materials," Transactions of the Faraday Society, vol. 57, pp. 829-838, 1961.

5. S. H. C. Lu and K. S. Pister, "Decomposition of deformation and representation of the free energy function for isotropic thermoelastic solids," International Journal of Solids and Structures, vol. 11, no. 7-8, pp. 927-934, 1975.

6. C. Sansour, "On the physical assumptions underlying the volumetric-isochoric split and the case of anisotropy," European Journal of Mechanics A/Solids, vol. 27, no. 1, pp. 28-39, 2008.

7. J. Lubliner, "A model of rubber viscoelasticity," Mechanics Research Communications, vol. 12, no. 2, pp. 93-99, 1985.

8. J. C. Simo and R. L. Taylor, "Quasi-incompressible finite elasticity in principal stretches. Continuum basis and numerical algorithms," Computer Methods in Applied Mechanics and Engineering, vol. 85, no. 3, pp. 273-310, 1991

9. R. W. Ogden, Non-linear Elastic Deformations, 1st ed. New York: Dover, 1997.

10. G. A. Holzapfel, Nonlinear Solid Mechanics: A Continuum Approach for Engineering, 1st ed. Chichester: John Wiley \& Sons, 2000.

11. D. W. Nicholson, "Tangent modulus matrix for finite element analysis of hyperelastic materials," Acta Mechanica, vol. 112, no. 1-4, pp. 187-201, 1995.

12. J. A. Weiss, B. N. Maker, and S. Govindjee, "Finite element implementation of incompressible, transversely isotropic hyperelasticity," Computer Methods in Applied Mechanics and Engineering, vol. 135, no. 1-2, pp. 107-128, 1996.

13. M. Itskov, "On the theory of fourth-order tensors and their applications in computational mechanics," Computer Methods in Applied Mechanics and Engineering, vol. 189, no. 2, pp. 419-438, 2000.

14. C. Suchocki, "A finite element implementation of Knowles stored-energy function: Theory, coding and applications," The Archive of Mechanical Engineering, vol. LVIII, no. 3, pp. 319-346, 2011. 
15. J. Cheng and L. T. Zhang, "A general approach to derive stress and elasticity tensors for hyperelastic isotropic and anisotropic biomaterials," International Journal of Computational Methods, vol. 15, no. 1, pp. 1850028 (1-33), 2018.

16. M. C. Boyce and E. M. Arruda, "Constitutive models of rubber elasticity: A review," Rubber Chemistry and Technology, vol. 73 , no. 3, pp. 504-523, 2000.

17. P. J. Blatz and W. L. Ko, "Application of finite elastic theory to the deformation of rubbery materials," Transactions of the Society of Rheology, vol. 6, no. 8, pp. 223-251, 1962.

18. R. J. Ogden, "Volume changes associated with the deformation of rubber-like solids," Journal of the Mechanics and Physics of Solids, vol. 24, no. 6, pp. 323-251, 1976.

19. I. Fried and A. R. Johnson, "A note on elastic energy density functions for largely deformed compressible rubber solids," Computer Methods in Applied Mechanics and Engineering, vol. 69, no. 1, pp. 53-64, 1988.

20. L. Anand, "A constitutive model for compressible elastomeric solids," Computational Mechanics, vol. 18, no. 5, pp. 339-355, 1996.

21. J. E. Bischoff, E. M. Arruda, and K. Grosh, "A new constitutive model for the compressibility of elastomers at finite deformations," Rubber Chemistry and Technology, vol. 74, no. 4, pp. 541-559, 2001.

22. J.-B. Le Cam, "A review of volume changes in rubbers: The effect of stretching," Rubber Chemistry and Technology, vol. 83, no. 3, pp. 247-269, 2010.

23. R. W. Penn, "Volume changes accompanying the extension of rubber," Transactions of the Society of Rheology, vol. 14, no. 4, pp. 509-517, 1970.

24. W. Ehlers and G. Eipper, "The simple tension problem at large volumetric strains computed from finite hyperelastic material laws," Acta Mechanica, vol. 130, no. 1-2, pp. 17-27, 1998.

25. F. Zhao, "Continuum constitutive modeling for isotropic hyperelastic materials," Advances in Pure Mathematics, vol. 6, no. 9, pp. 571-582, 2016.

26. T. J. R. Hughes, The Finite Element Method: Linear Static and Dynamic Finite Element Analysis, 1st ed. Englewood Cliffs, NJ: Prentice-Hall, 1987.

27. O. C. Zienkiewicz, R. L. Taylor, and J. Z. Zhu, The Finite Element Method: Its Basis and Fundamentals, 6th ed. New York: Elsevier Butterworth-Heinemann, 2005.

28. K.-J. Bathe, Finite Element Procedures, 2nd ed. Watertown, MA: Klaus-Jürgen Bathe, 2014.

29. F. Zhao, "Anisotropic continuum stored energy functional solved by Lie group and differential geometry," Advances in Pure Mathematics, vol. 8, no. 7, pp. 631-651, 2018.

30. O. Kintzel and Y. Başar, "Fourth-order tensors-tensor differentiation with applications to continuum mechanics. Part I: Classical tensor analysis," Zeitschrift für angewandte Mathematik und Mechanik, vol. 86, no. 4, pp. 291-311, 2006.

31. O. Kintzel, "Fourth-order tensors-tensor differentiation with applications to continuum mechanics. Part II: Tensor analysis on manifolds," Zeitschrift für angewandte Mathematik und Mechanik, vol. 86, no. 4, pp. 312-334, 2006.

32. B. Storåkers, "On material representation and constitutive branching in finite compressible elasticity," Journal of the Mechanics and Physics of Solids, vol. 34, no. 2, pp. 125-145, 1986.

33. F. Zhao, "On constitutive modeling of arteries," Journal of Advances in Applied Mathematics, vol. 4, no. 2, pp. 54-68, 2019.

34. O. Starkova and A. Aniskevich, "Poisson's ratio and the incompressibility relation for various strain measures with the example of a silica-filled SBR rubber in uniaxial tension tests," Polymer Testing, vol. 29, no. 3, pp. 310-318, 2010.

35. C. O. Horgan and J. G. Murphy, "Constitutive modeling for moderate deformations of slightly compressible rubber," Journal of Rheology, vol. 53, no. 1, pp. 153-168, 2009.

36. A. F. Amin, M. S. Alam, and Y. Okui, "Measurement of lateral deformation in natural and high damping rubbers in large deformation uniaxial tests," Journal of Testing and Evaluation, vol. 31, no. 6, pp. 524-532, 2003.

37. P. Charrier, B. Dacorohna, B. Hanouzet, and P. Laborde, "An existence theorem for slightly compressible materials in nonlinear elasticity," SIAM Journal on Mathematical Analysis, vol. 19, no. 1, pp. 70-85, 1988.

38. J. G. Murphy and G. A. Rogerson, "Modelling slight compressibility for hyperelastic anisotropic materials," Journal of Elasticity, vol. 131, no. 2, pp. 171-181, 2018.

39. F. Zhao, "The continuum stored energy for constitutive modeling finite deformations of polymeric materials," Advances in Pure Mathematics, vol. 7, no. 10, pp. 597-613, 2017.

40. Z. P. Bažant, M. Gattu, and J. Vorel, "Work conjugacy error in commercial finite-element codes: its magnitude and how to compensate for it," Proceedings of the Royal Society A, vol. 468, pp. 3047-3058, 2012.

41. W. Ji, A. M. Waas, and Z. P. Bažant, "Errors caused by non-work-conjugate stress and strain measures and necessary corrections in finite element programs," Journal of Applied Mechanics T-ASME, vol. 77, no. 4, pp. 044504 (1-5), 2010.

42. — - "On the importance of work-conjugacy and objective stress rates in finite deformation incremental finite element analysis," Journal of Applied Mechanics T-ASME, vol. 80, no. 4, pp. 041024 (1-9), 2013. 